\title{
Validity and reliability of the Norwegian translation of the Achilles tendon Total Rupture Score
}

\author{
Ståle Bergman Myhrvold ${ }^{1}$ - Øystein Sandnes ${ }^{2} \cdot$ Sigurd Erik Hoelsbrekken $^{3}$
}

Received: 10 May 2017 / Accepted: 16 August 2017 / Published online: 19 August 2017

(C) The Author(s) 2017. This article is an open access publication

\begin{abstract}
Purpose Patient reported outcome measures (PROMs) are increasingly being used in clinical research. The Achilles tendon Total Rupture Score (ATRS) is a PROM designed to assess outcomes in patients with ruptures of the Achilles tendon. The aim of this study was to develop a Norwegian adaption of the ATRS and evaluate its validity and reliability.

Methods The ATRS was translated into Norwegian in accordance with recommended guidelines. The study included 94 patients treated for Achilles tendon ruptures from January 2010 until February 2013, and the web-based questionnaires were administered twice. Reliability was assessed by Cronbach's alpha, the intraclass correlation coefficient (ICC) and minimal detectable change (MDC). Construct validity was evaluated by calculating the Spearman's correlation coefficient with the five subclasses of the Foot and Ankle Outcome Score (FAOS), the SF-36 subclass physical function and the SF-36 physical and mental summary scores.
\end{abstract}

Ståle Bergman Myhrvold

stalemyhrvold@gmail.com; samh@ahus.no

Øystein Sandnes

oys.sandnes@gmail.com

Sigurd Erik Hoelsbrekken

s.e.hoelsbrekken@medisin.uio

1 Department of Orthopedics, Akershus University Hospital, 1478 Lørenskog, Norway

2 Department of Orthopedics, Ringerike Hospital, 3511 Hønefoss, Norway

3 Department of Orthopedic and Rheumatic Surgery, Kongsvinger Hospital, P.O. Box 901, 2226 Kongsvinger, Norway
Results Both internal consistency (Cronbach's alpha $=0.96)$ and test-retest reliability $(\mathrm{ICC}=0.90)$ were excellent, and the MDC was 2.12 at the group level and 16.98 at the individual level. Construct validity was supported by Spearman's correlation coefficients above 0.7 with the SF-36 subclass physical function and the SF-36 physical summary score as well as the FAOS subclasses Sport/Rec and quality of life. There was no floor effect, and 6 patients (6.4\%) achieved a maximum score of 100.

Conclusions The Norwegian adaption of the ATRS demonstrates acceptable validity and reliability for use in the Norwegian population to assess clinical outcomes in patients with Achilles tendon ruptures.

Keywords ATRS · Achilles tendon rupture - Crosscultural $\cdot$ Norwegian $\cdot$ Reliability $\cdot$ Validity

$\begin{array}{ll}\text { Abbreviations } \\ \text { PROM } & \text { Patient reported outcome measure } \\ \text { ATRS } & \text { Achilles tendon Total Rupture Score } \\ \text { ICC } & \text { Intraclass correlation coefficient } \\ \text { FAOS } & \text { Foot and Ankle Outcome Score } \\ \text { SF-36 } & \text { Short Form-36 version } 2 \\ \text { KOOS } & \text { Knee injury and Osteoarthritis Outcome } \\ & \text { Score } \\ \text { PCS } & \text { Physical component summary of the SF-36 } \\ \text { MCS } & \text { Mental component summary of the SF-36 } \\ \text { PF } & \text { Physical Function Score of the SF-36 } \\ \text { Sport/Rec } & \text { Function of sport and recreation subclass in } \\ & \text { FAOS } \\ \text { ADL } & \text { Function in daily living } \\ \text { QOL } & \text { Foot- and ankle-related quality of life subclass } \\ & \text { in FAOS } \\ \text { SEM } & \text { Standard error of measurement }\end{array}$




\section{SD Standard deviation \\ MDC Minimal detectable change}

\section{Introduction}

An acute rupture of the Achilles tendon represents a common injury [8], but the best choice of treatment remains controversial [6]. Recent studies emphasizing early mobilization have reported improved results after non-operative treatment [17], underlining the need to evaluate outcomes beyond occurrences of common complications such as reruptures, wound healing problems, infections and nerve injuries. Patient reported outcome measures (PROMs) are questionnaires answered by the patients themselves, and they are becoming increasingly popular when evaluating treatment results and patients satisfaction in clinical studies. The acute Achilles tendon Rupture Score (ATRS) is a PROM developed to assess outcomes in patients who have undergone treatment for acute Achilles tendon ruptures [13], and it has been validated in Swedish, English, Danish, Turkish, Persian, Dutch, Brazilian Portuguese and Italian [1, 4, 7, 9, 13, 15, 19, 21]. There are presently no PROMs in Norwegian validated for assessing outcomes after Achilles tendon injuries, and the purpose of this study was to translate and validate a Norwegian version of the ATRS. This will facilitate future research on the treatment of Achilles tendon ruptures in the Norwegian population.

\section{Materials and methods}

Patients were identified by searching the hospitals electronic admission record using the code for Achilles tendon rupture (S86.0) from the international classification of diseases, 10th revision (ICD-10). Patients aged 18-60 treated for acute ruptures of the Achilles tendon from 2010 until 2013 were considered eligible for inclusion, and a total of 155 patients gave consent for participation in the study (Fig. 1). There is no consensus regarding sample size calculations for the validation of PROMs, but we adhered to a recommended minimum of 50 patients [18]. The questionnaires ATRS, Foot and Ankle Outcome Score (FAOS) and the 36-Item Short Form Health Survey (SF-36) version 2 were completed online by logging on to a secured server. Patients who completed the questionnaires incorrectly were excluded from the study. Patients, who failed to complete the second set of questionnaires within four to eight weeks of completing the first set of questionnaires, were excluded from test-retest analysis. Patients reporting a change in their condition between completing the two sets of questionnaires were also excluded from test-retest analysis.

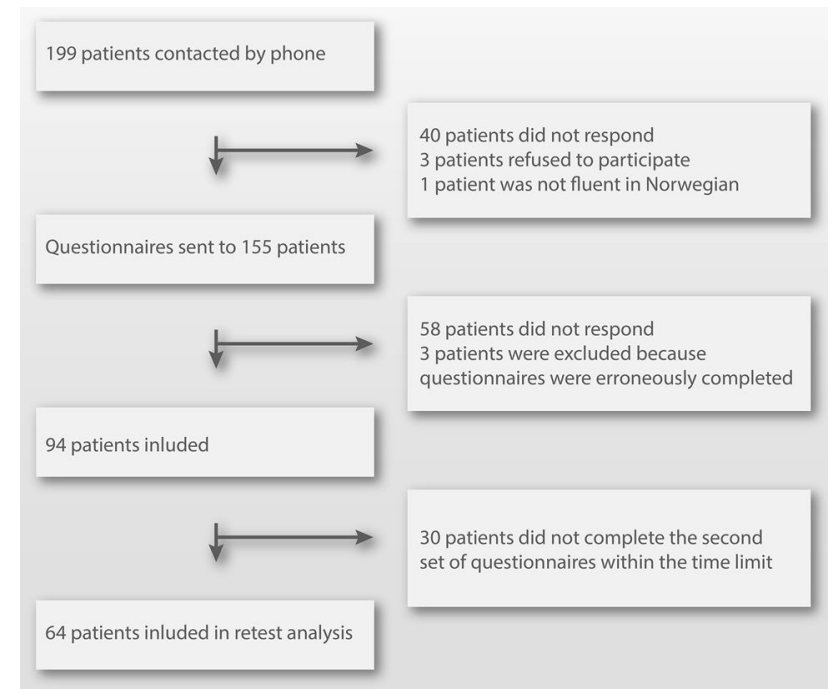

Fig. 1 Inclusion flow chart

\section{Outcome measures and translations}

The ATRS questionnaire contains ten questions, and each question is answered on an 11-point Likert scale ranging from 0 to 10 . The total score is calculated by summing the individual Likert items. A score of 100 represents the absence of symptoms, whereas a score of 0 represents severe symptoms. The ATRS was translated into Norwegian according to recommended guidelines [2]. Three independent translators performed the translation from Swedish to Norwegian, and discrepancies were resolved by discussion. Two independent translators fluent in Norwegian and Swedish performed the back-translation into Swedish. The Norwegian language is very similar to Swedish, and the Norwegian version was reviewed and approved by the developer of the original Swedish ATRS.

The SF-36 is a self-assessment health status questionnaire composed of 36 questions sorted into eight multi-item scales. The SF-36 also provides two summarized measures represented by the physical component summary (PCS) and the mental component summary (MCS) [20]. The validity and reliability of the Norwegian translation of SF-36 have been found to be satisfactory $[11,12]$.

The FAOS questionnaire is a modification of the Knee injury and Osteoarthritis Outcome Score (KOOS). The only difference between KOOS and FAOS is the replacement of "knee" in KOOS with "foot/ankle" in FAOS [16]. FAOS consists of 42 questions divided into five subscales: pain, symptoms, function in daily living (ADL), function in sport and recreation (Sport/Rec) and foot- and ankle-related quality of life (QOL). Each subscale contains questions answered on a 5-point Likert scale ranging from 0 to 4 . A normalized score (100 indicating no symptoms and 0 indicating severe 
symptoms) is calculated for each subscale. By replacing "knee" with "foot/ankle", a Norwegian translation of FAOS from the Norwegian adaptation of KOOS in accordance with the original version of FAOS [16] was created.

\section{Reliability}

Internal consistency indirectly evaluates whether different items in a questionnaire produce similar scores by measuring the correlation between the items. Poor internal consistency indicates the lack of correlation, which invalidates the creation of a summarized score. Cronbach's alpha was used to evaluate internal consistency, and a Cronbach's alpha greater than 0.7 was considered acceptable [3].

Test-retest reliability was defined as the ability of the questionnaires to measure the same outcome twice, and the ATRS, FAOS and SF-36 were completed at two different occasions with a washout period of four to eight weeks. The mean (SD) time between injury and completion of the questionnaires was 54.6 months (8.9) with a range of 36.1-72.7 months.

Test-retest reliability was calculated by the intraclass correlation coefficient (ICC) using a two-way random model, agreement and average measure (ICC 2.k). ICC was interpreted as follows: excellent $(>0.75)$, fair to good $(0.40-0.75)$ and poor $(<0.40)$ [5].

Standard error of measurement (SEM) is the standard deviation of an observed test score, and there is a 95\% probability that the persons "true" score is within $\pm 2 \times$ SEM of the observed score. Minimal detectable change (MDC) represents the smallest amount of change that can be detected beyond measurement error. SEM was calculated using the formula: standard deviation (SD) $\times \sqrt{ }(1-$ ICC). MDC at the individual level was calculated by $1.96 \times \sqrt{ } 2 \times$ SEM and at the group level by $(1.96 \times \sqrt{ } 2 \times \mathrm{SEM}) / \sqrt{ } n$.

\section{Construct validity}

We evaluated criterion validity of the ATRS questionnaire by testing for correlations with the SF-36 component summaries PCS and MCS in addition to the subscale physical function (PF). We also calculated correlations between the ATRS and the five subscales of FAOS. Correlations were evaluated by use of the Spearman's rank correlation coefficient as it is more robust to skewed data and outliers compared to Pearson correlation coefficient. Construct validity for ATRS was defined by hypothesizing a priori, correlation with SF-36 PF, SF-36 PCS, FAOS symptoms, FAOS Sport/ Rec and FAOS QOL equal to or above 0.7 , based on results from the Danish and Swedish validation studies [7, 13].

\section{Floor and ceiling effects}

The presence of floor or ceiling effect was defined by more than $15 \%$ of the responders achieving the lowest or highest possible score, respectively [18].

\section{Ethics}

The study was approved by the Regional Committee for Medical and Health Research Ethics of Norway (reference no. 2015/974).

\section{Statistical analysis}

Categorical data were compared using the Chi-squared test. The Shapiro-Wilk test and inspection of histograms were used to test for normality, and the Levene's test was used to assess equality of variances. Continuous variables showing normal distribution were presented with mean and SD and compared using the student $t$ test or analysis of variance (ANOVA). Variables showing a non-normal distribution were presented with median and range. All analysis was performed in SPSS Statistics for Macintosh, Version 24.0 (Armonk, NY: IBM Corp).

\section{Results}

\section{Demographics}

Patient characteristics were comparable for non-responders and responders, and there were no significant differences between the groups (Table 1).

Table 1 Patient characteristics

\begin{tabular}{lccc}
\hline & $\begin{array}{l}\text { Non-responders } \\
N=105\end{array}$ & Responders $N=94$ & Retest ATRS $N=64$ \\
\hline Male sex (\%) & $79(75.2)$ & $71(75.5)$ & $48(75.0)$ \\
Mean age in years (SD) & $45.3(8.5)$ & $46.7(8.5)$ & $46.8(8.5)$ \\
Operative treatment (\%) & $92(87.6)$ & $89(94.7)$ & $61(95.3)$ \\
$\begin{array}{l}\text { Mean time between injury and } \\
\text { completion of the questionnaires } \\
\text { in months (SD) }\end{array}$ & $54.3(9.2)$ & $54.9(9.1)$ & $54.5(8.3)$ \\
\hline
\end{tabular}




\section{Translation}

Norwegian and Swedish are closely related languages, and cultural differences are small. There was a single discrepancy in the forward translation of the ATRS regarding the translation of "Are you limited". There are two different expressions that can be used interchangeably in Norwegian, and we chose the expression that most closely resembles the wording in the original Swedish version and that is also being used by the Norwegian translation of SF-36. There were no discrepancies in the back-translation of the ATRS.

\section{Reliability}

The median (range) time between completion of the two set of questionnaires was 42 (28-56) days. Internal consistency for ATRS was 0.96, ICC was 0.90, and the SEM was 6.13. The MDC was 16.98 at the individual level and 2.12 at the group level.

\section{Validity}

The ATRS demonstrated satisfactory correlations with the SF-36 component summary PCS and the SF-36 subscale PF as well as the FAOS subscales QOL and Sport/Rec, as defined by Spearman's rank correlation coefficients above 0.7 (Table 2).

\section{Floor and ceiling effects}

There was no floor effect observed for ATRS, and 6 patients (6.4\%) achieved a score of 100 . In contrast, the SF-36 subscale PF and several of the FAOS subscales displayed high ceiling effects (Table 3 ).

\section{Discussion}

The main finding of the study was acceptable validity and reliability demonstrated by the Norwegian adaptation of the

Table 2 Criterion validity of the ATRS assessed by Spearman's rank correlation coefficient

\begin{tabular}{lll}
\hline & ATRS & $p$ value \\
\hline SF-36 PCS & 0.72 & $<0.0001$ \\
SF-36 PF & 0.71 & $<0.0001$ \\
SF-36 MCS & 0.11 & n.s. \\
FAOS Sport/Rec & 0.81 & $<0.0001$ \\
FAOS QOL & 0.76 & $<0.0001$ \\
FAOS ADL & 0.66 & $<0.0001$ \\
FAOS pain & 0.64 & $<0.0001$ \\
FAOS symptom & 0.61 & $<0.0001$ \\
\hline
\end{tabular}

Table 3 Median values and ceiling effects

\begin{tabular}{lcl}
\hline & Median (range) & Highest score $n(\%)$ \\
\hline ATRS & $87(16.0-100)$ & $6(6.4)$ \\
SF-36 PCS & $55.9(30.2-62.8)$ & $0(0)$ \\
SF-36 MCS & $55.5(36.2-68.4)$ & $0(0)$ \\
SF-36 PF & $87.5(12.5-100)$ & $45(47.9)$ \\
FAOS Sport/Rec & $90.0(5.0-100)$ & $32(34.0)$ \\
FAOS QOL & $87.5(12.5-100)$ & $26(27.7)$ \\
FAOS ADL & $98.5(33.8-100)$ & $44(46.8)$ \\
FAOS pain & $97.2(33.3-100)$ & $43(45.8)$ \\
FAOS symptom & $89.3(35.7-100)$ & $20(21.3)$ \\
\hline
\end{tabular}

ATRS. The Norwegian translation of the ATRS can therefore be used in the Norwegian population to assess clinical results in patients sustaining ruptures of the Achilles tendon.

The observed internal consistency was comparable to previous reports from other cross-cultural adaptations of the ATRS ranging from 0.89 to $0.97[1,4,7,9,13,15,19]$. The SEM was also in agreement with previously reported results ranging from 3.2 to 10.91 [1, 4, 7, 9, 15]. The MDC of 16.98 at the individual level was considerably lower than 30.24 reported by the Dutch validation study [15], but similar to 18.5 observed in the Danish study [7]. The MDC of 2.12 at the group level was lower compared to the Dutch and English adaptations with 3.49 and 6.75, respectively [4, 15]. To our knowledge, MDC has only been reported by the Danish, Dutch and English validation studies. Based on the MDC values, the Norwegian translation of the ATRS is well suited to compare groups of patients as a difference above 2.12 points reflects a real change, but the individual MDC of 16.98 restricts its usefulness in the follow-up of individual patients. The ICC was similar to 0.908 attained in the Danish validation and well within what has previously been reported (0.852-0.986) [1, 7, 13, 15, 19].

There are no PROMs validated in Norwegian for assessing clinical outcomes after Achilles tendon ruptures, and the Norwegian adaptation of the ATRS could not be correlated with an outcome measure specific for Achilles tendon ruptures. We therefore correlated the ATRS with SF-36 domains equivalent to the Danish adaptation [7]. We also tested for correlations with all five FAOS subscales similar to the Swedish validation study [13]. The correlation data attained in the present study were similar to the results from the Swedish and Danish validations $[7,13]$. This was in accordance with expectations since Danish, Norwegian and Swedish are closely related languages, and cultural differences between the countries are small. The correlation coefficients between the Norwegian ATRS and the SF-36 PCS and SF-36 PF were all above 0.7 , as hypothesized. The correlation coefficients were also above 0.7 with the FAOS subscales 
QOL and Sport/Rec. Although the correlation coefficient was below 0.7 with the FAOS subscale symptoms, four out of five a priori hypothesized correlations were confirmed by the study, which is considered acceptable [18].

None of the patients attained an ATRS score of 0 , and thus, no floor effect was present. A ceiling effect was observed in $6.4 \%$ of the patients, but this was below the pre-defined threshold of $15 \%$. In contrast, the SF-36 subscale PF and all the FAOS subscales except for symptoms demonstrated ceiling effects well above $15 \%$. Unfortunately, neither the Dutch, Swedish or Turkish validation study reported ceiling data for the FAOS subscales [9, 13, 15], but such high ceiling effects do question the suitability of the Norwegian adaptation of FAOS to assess clinical results in patients sustaining Achilles tendon ruptures. We did not evaluate sensitivity to changes over time for the Norwegian ATRS, but responsiveness has been evaluated for the original version $[4,10]$.

The time period between the two test occasions was longer in the present study compared to previous ATRS adaptations, which may potentially have influenced reliability testing. However, the mean time from injury to answering the first questionnaire was 54.9 months in the present cohort, and only minor clinical improvements can be expected more than one year after injury [14]. This indicates that the cohort was fairly homogenous with respect to state of rehabilitation, and results from test-retest analysis were similar to what has previously been reported.

Only 94 out of 199 patients (47\%) eligible for inclusion completed the first set of questionnaires. The low response rate may have introduced selection bias, and we are unable to account for the non-responders apart from characteristics provided in Table 1. It is therefore difficult to ascertain that the included group of patients is representative of the general population. Selection bias may also have been introduced in test-retest analysis as only 64 out of 94 patients (68\%) completed the second set of questionnaires.

The results from this study are similar to previous adaptations of the ATRS, and both the validity and reliability of the Norwegian ATRS were acceptable. This allows for follow-up and comparison of different treatment options using a PROM adapted to the Norwegian language that is designed to evaluate results after Achilles tendon ruptures. It will also facilitate future research on the treatment of Achilles tendon ruptures in the Norwegian population.

\section{Conclusion}

The Norwegian adaptation of the ATRS demonstrates acceptable validity and reliability for use in the Norwegian population to assess the clinical outcome in patients with Achilles tendon ruptures.

Authors' contribution SM contributed to the design of the study, collection and analysis of data and drafting and revising the manuscript. ØS contributed to the design of the study, collection of data and revising the manuscript. SEH contributed to the design of the study, collection and analysis of data and drafting and revising the manuscript.

\section{Compliance with Ethical Standards}

Conflict of interest The authors declare that they have no conflict of interest.

Funding The project was funded by $\mathrm{PhD}$ grants from Helse $\mathrm{S} \emptyset \mathrm{r}-\emptyset_{\mathrm{st}}$ Regional Health Company.

Ethical approval The study was approved by the Regional Committee for Medical and Health Research Ethics of Norway (reference no. 2015/974).

Informed consent Informed consent for participation was collected by phone. The patients activation of their electronic link leading to logging on to a secured server was accepted as digital signing of informed consent.

Open Access This article is distributed under the terms of the Creative Commons Attribution 4.0 International License (http://creativecommons.org/licenses/by/4.0/), which permits unrestricted use, distribution, and reproduction in any medium, provided you give appropriate credit to the original author(s) and the source, provide a link to the Creative Commons license, and indicate if changes were made.

\section{References}

1. Ansari NN, Naghdi S, Hasanvand S, Fakhari Z, Kordi R, NilssonHelander K (2016) Cross-cultural adaptation and validation of Persian Achilles tendon Total Rupture Score. Knee Surg Sports Traumatol Arthrosc 24:1372-1380

2. Beaton DE, Bombardier C, Guillemin F, Ferraz MB (2000) Guidelines for the process of cross-cultural adaptation of self-report measures. Spine (Phila Pa 1976) 25:3186-3191

3. Bland JM, Altman DG (1997) Cronbach's alpha. BMJ 314:572

4. Carmont MR, Silbernagel KG, Nilsson-Helander K, Mei-Dan O, Karlsson J, Maffulli N (2013) Cross cultural adaptation of the Achilles tendon Total Rupture Score with reliability, validity and responsiveness evaluation. Knee Surg Sports Traumatol Arthrosc 21:1356-1360

5. Cicchetti DV (1994) Guidelines, criteria, and rules of thumb for evaluating normed and standardized assessment instruments in psychology. Psychol Assess 6:284-290

6. Erickson BJ, Mascarenhas R, Saltzman BM, Walton D, Lee $\mathrm{S}$, Cole BJ et al (2015) Is operative treatment of Achilles tendon ruptures superior to nonoperative treatment?: a systematic review of overlapping meta-analyses. Orthop J Sports Med 3:2325967115579188

7. Ganestam A, Barfod K, Klit J, Troelsen A (2013) Validity and reliability of the Achilles tendon total rupture score. J Foot Ankle Surg 52:736-739 
8. Jarvinen TA, Kannus P, Maffulli N, Khan KM (2005) Achilles tendon disorders: etiology and epidemiology. Foot Ankle Clin $10: 255-266$

9. Kaya Mutlu E, Celik D, Kilicoglu O, Ozdincler AR, NilssonHelander K (2015) The Turkish version of the Achilles tendon Total Rupture Score: cross-cultural adaptation, reliability and validity. Knee Surg Sports Traumatol Arthrosc 23:2427-2432

10. Kearney RS, Achten J, Lamb SE, Parsons N, Costa ML (2012) The Achilles tendon total rupture score: a study of responsiveness, internal consistency and convergent validity on patients with acute Achilles tendon ruptures. Health Qual Life Outcomes 10:24

11. Kopjar B (1996) The SF-36 health survey: a valid measure of changes in health status after injury. Inj Prev 2:135-139

12. Loge JH, Kaasa S, Hjermstad MJ, Kvien TK (1998) Translation and performance of the Norwegian SF-36 Health Survey in patients with rheumatoid arthritis. I. Data quality, scaling assumptions, reliability, and construct validity. J Clin Epidemiol 51:1069-1076

13. Nilsson-Helander K, Thomee R, Silbernagel KG, Thomee P, Faxen E, Eriksson BI et al (2007) The Achilles tendon Total Rupture Score (ATRS): development and validation. Am J Sports Med $35: 421-426$

14. Olsson N, Nilsson-Helander K, Karlsson J, Eriksson BI, Thomee R, Faxen E et al (2011) Major functional deficits persist 2 years after acute Achilles tendon rupture. Knee Surg Sports Traumatol Arthrosc 19:1385-1393

15. Opdam KT, Zwiers R, Wiegerinck JI, Kleipool AE, Haverlag R, Goslings JC et al (2016) Reliability and validation of the Dutch
Achilles tendon Total Rupture Score. Knee Surg Sports Traumatol Arthrosc. doi:10.1007/s00167-016-4242-7

16. Roos EM, Brandsson S, Karlsson J (2001) Validation of the foot and ankle outcome score for ankle ligament reconstruction. Foot Ankle Int 22:788-794

17. Soroceanu A, Sidhwa F, Aarabi S, Kaufman A, Glazebrook M (2012) Surgical versus nonsurgical treatment of acute Achilles tendon rupture: a meta-analysis of randomized trials. J Bone Joint Surg Am 94:2136-2143

18. Terwee CB, Bot SD, de Boer MR, van der Windt DA, Knol DL, Dekker J et al (2007) Quality criteria were proposed for measurement properties of health status questionnaires. J Clin Epidemiol 60:34-42

19. Vascellari A, Spennacchio P, Combi A, Grassi A, Patella S, Bisicchia $S$ et al (2016) Cross-cultural adaptation and multi-centric validation of the Italian version of the Achilles tendon Total Rupture Score (ATRS). Knee Surg Sports Traumatol Arthrosc. doi:10.1007/s00167-016-4152-8

20. Ware JE Jr, Gandek B (1998) Overview of the SF-36 Health Survey and the International Quality of Life Assessment (IQOLA) project. J Clin Epidemiol 51:903-912

21. Zambelli R, Pinto RZ, Magalhaes JM, Lopes FA, Castilho RS, Baumfeld D et al (2016) Development of the Brazilian Portuguese version of the Achilles Tendon Total Rupture Score (ATRS BrP): a cross-cultural adaptation with reliability and construct validity evaluation. BMC Sports Sci Med Rehabil 8:11 